\title{
Combination Cancer Immunotherapy in Hepatocellular Carcinoma
}

\author{
Masatoshi Kudo \\ Department of Gastroenterology and Hepatology, Kindai University Faculty of Medicine, \\ Osaka-Sayama, Japan
}

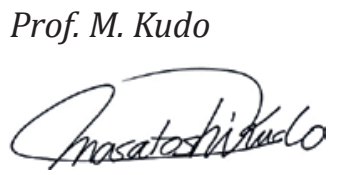

Editor Liver Cancer

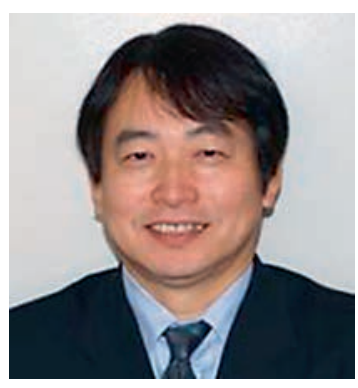

\section{Introduction}

Combination cancer immunotherapy is becoming a major topic in cancer therapy research including in hepatocellular carcinoma (HCC). Well-known combinations include two types of immune checkpoint inhibitors (anti-PD-1/PD-L1 and anti-CTLA-4 antibodies), anti-PD-1/ PD-L1 antibody with a molecular targeted agent, and anti-PD-1/PD-L1 or -CTLA-4 antibody with existing locoregional therapies (Fig. 1).

Immunotherapy for the treatment of various types of cancer has advanced rapidly in recent years. The Federal Drug Administration (FDA) designated nivolumab, an anti-PD-1 antibody, as a breakthrough therapy in 2014, and pembrolizumab, another anti-PD-1 antibody, also received this designation. Nivolumab was approved as a highly effective agent for the treatment of certain malignancies, including malignant melanoma, non-small cell lung cancer, kidney cancer, Hodgkin lymphoma, head and neck cancer, and gastric cancer [1-6]. Promising clinical trials evaluating this agent for the treatment of many other types of cancer are currently ongoing. HCC is more heterogeneous than other types of solid cancer and hematological malignancies, and is not associated with a specific driver mutation. HCC cannot be treated with agents that impair liver function, and thus requires different therapeutic strategies than those used for other cancers. Despite this limitation, the CheckMate 040 study revealed that nivolumab is a promising therapy for HCC [7]. Many pharmaceutical companies started phase III or earlier-phase trials of anti-PD-1/PD-L1 antibodies for HCC treatment (Table 1). In addition, the FDA approved nivolumab for second-line therapy after sorafenib in September 2017. 


\section{Liver Cancer}

\begin{tabular}{l|l}
\hline Liver Cancer 2018;7:20-27 \\
\hline DOI: 10.1159/000486487 & $\begin{array}{l}\text { (c) 2018 S. Karger AG, Basel } \\
\text { www.karger.com/lic }\end{array}$ \\
\hline
\end{tabular}

Kudo: Combination Cancer Immunotherapy in Hepatocellular Carcinoma

Table 1. Immune checkpoint inhibitors in hepatocellular carcinoma clinical trials

\begin{tabular}{|c|c|c|c|c|c|c|}
\hline Target cell & $\begin{array}{l}\text { Target } \\
\text { molecule }\end{array}$ & Development code & Drug name & $\begin{array}{l}\text { Commercial } \\
\text { name }\end{array}$ & Antibody & Company \\
\hline \multirow[t]{2}{*}{ T lymphocyte } & PD-1 & $\begin{array}{l}\text { BMS-36558 } \\
\text { ONO-4538 }\end{array}$ & Nivolumab & Optivo & $\begin{array}{l}\text { Fully human } \\
\text { IgG4 antibody }\end{array}$ & ONO/BMS \\
\hline & PD-1 & MK-4375 & Pembrolizumab & Keytruda & $\begin{array}{l}\text { Humanized } \\
\text { IgG4 antibody }\end{array}$ & Merck \\
\hline \multirow[t]{3}{*}{ Tumor cell } & PD-L1 & MPDL3280A & Atezolizumab & Tecentriq & $\begin{array}{l}\text { Fully humanized } \\
\text { IgG1 antibody }\end{array}$ & Roche \\
\hline & PD-L1 & MEDI4736 & Durvalumab & Imfinzi & $\begin{array}{l}\text { Humanized } \\
\text { IgG1 antibody }\end{array}$ & AstraZeneca \\
\hline & PD-L1 & MSB-0010718C & Avelumab & Bavencio & $\begin{array}{l}\text { Humanized } \\
\text { IgG1 antibody }\end{array}$ & Merck Serono \\
\hline \multirow[t]{2}{*}{ T lymphocyte } & CTLA-4 & BMS-734016 & Ipilimumab & Yervoy & $\begin{array}{l}\text { Fully humanized } \\
\text { IgG1 antibody }\end{array}$ & BMS Medarex \\
\hline & CTLA-4 & MEDI1123 & Tremelimumab & $\begin{array}{l}\text { Not yet } \\
\text { approved }\end{array}$ & $\begin{array}{l}\text { Fully humanized } \\
\text { IgG2 antibody }\end{array}$ & $\begin{array}{l}\text { AstraZeneca } \\
\text { MedImmune }\end{array}$ \\
\hline
\end{tabular}

Combination with locoregional therapy or other immune checkpoint inhibitors/TKIs.

Clinical trials of some have already started.

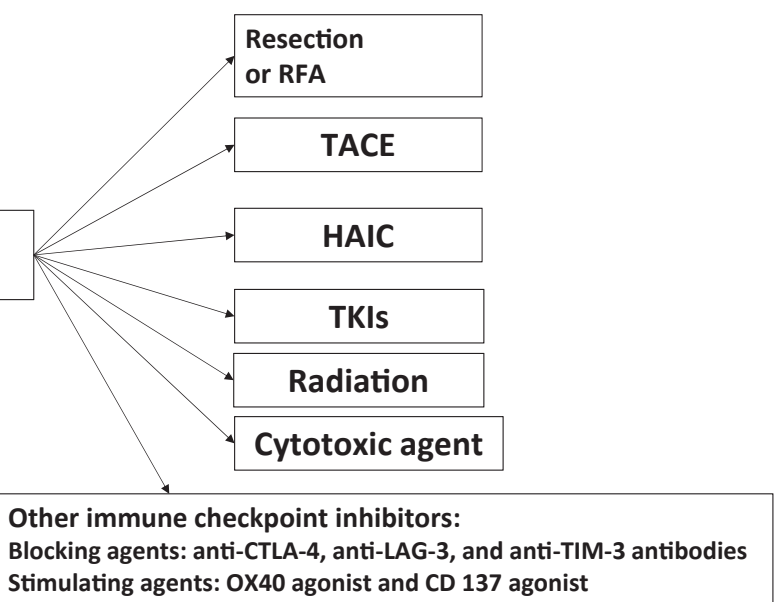

Fig. 1. Treatment strategy using immune checkpoint inhibitors. Future direction: combination therapy. RFA, radiofrequency ablation; TACE, transarterial chemoembolization; HAIC, hepatic arterial infusion chemotherapy; TKIs, tyrosine kinase inhibitors.

\section{Combination of Immune Checkpoint Inhibitors}

Anti-PD-1/PD-L1 and anti-CTLA-4 antibodies are expected to be promising agents in HCC immunotherapy, and clinical trials evaluating the simultaneous blockade of multiple immune checkpoints are currently ongoing (Fig. 1; Table 2). The high efficacy of combination therapy was demonstrated in malignant melanoma [6], and a trial of the same combination for the treatment of HCC is currently ongoing [8]. Inhibition of the PD-1/PD-L1 pathway will not activate tumor immunity as expected if the required $\mathrm{CD} 8^{+} \mathrm{T}$ cells are not present in the cancer tissue. However, simultaneous inhibition of the B7-CTLA-4 pathway by an anti-CTLA-4 antibody can increase the number of activated $\mathrm{CD}^{+} \mathrm{T}$ cells in lymph nodes, followed by an 


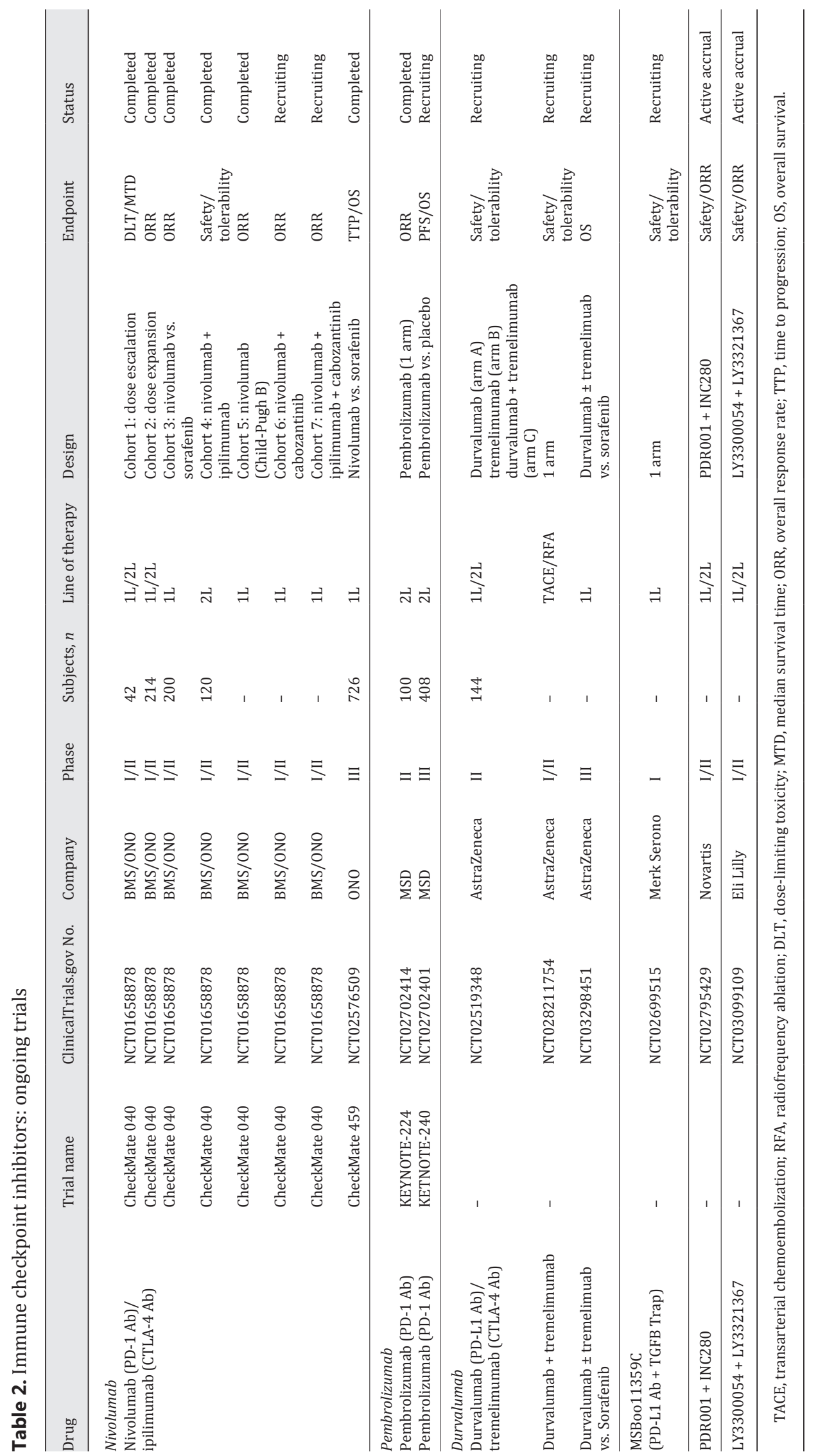




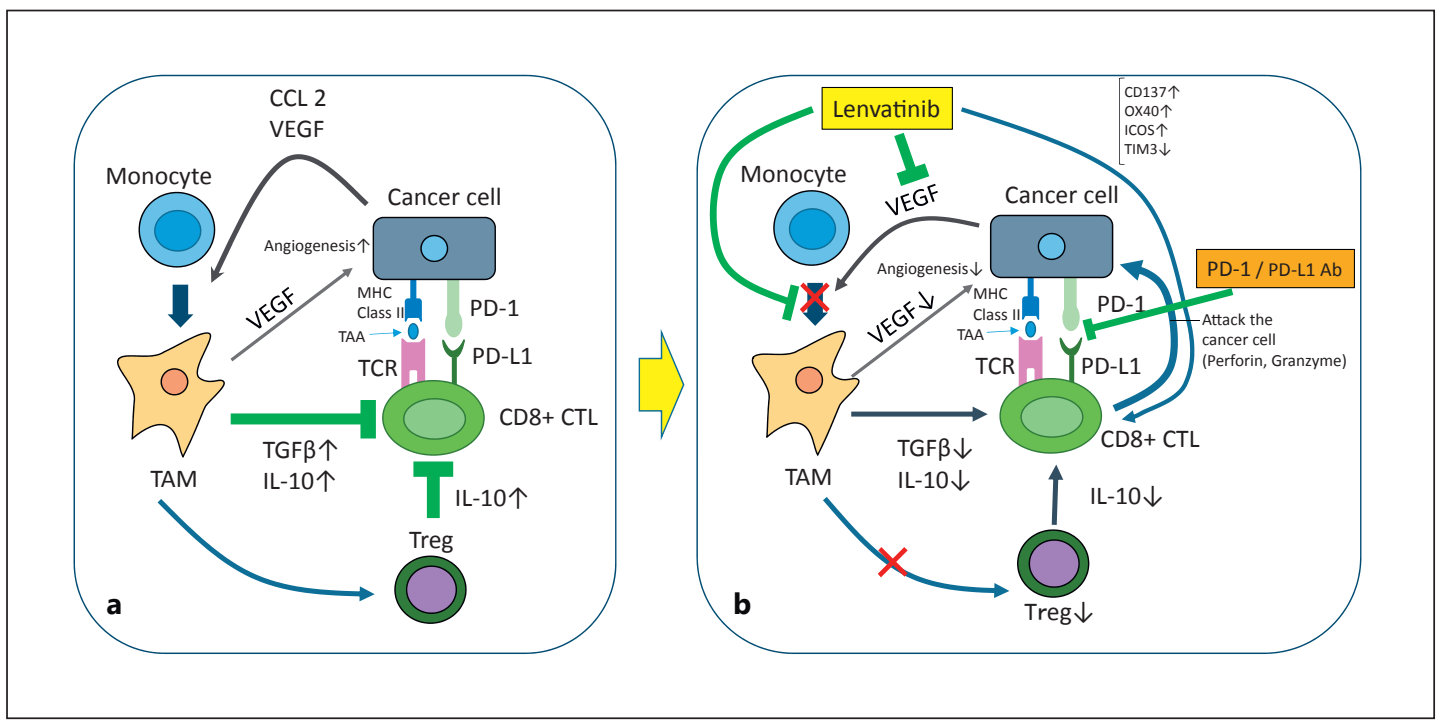

Fig. 2. Mechanism of synergistic effects of lenvatinib and anti-PD-1/PD-L1 antibodies. a Immunosuppressive microenvironment + PD-1/PD-L1 immunosuppression. $\mathbf{b}$ Lenvatinib + anti-PD-1/PD-L1 antibodies synergistically induce PD-1/PD-L1 blockade and inhibit the immunosuppressive microenvironment.

increase in the number of activated $\mathrm{CD}^{+} \mathrm{T}$ cells infiltrating into tumor tissues, thereby enhancing the antitumor effects. In addition, anti-CTLA-4 antibody therapy may be effective against regulatory $\mathrm{T}$ cells in the cancer immunosuppressive microenvironment.

This is the rationale for the use of combination therapy with an anti-PD-1/PD-L1 antibody and an anti-CTLA-4 antibody, and several trials evaluating these combinations for the treatment of HCC are currently ongoing (Table 2). The CheckMate 040 study tested the efficacy of nivolumab in combination with ipilimumab at varying doses and dose intervals. Another trial comparing the efficacy and safety of combination therapy with durvalumab (anti-PD-L1 antibody) plus tremelimumab (anti-CTLA-4 antibody) to those of monotherapy is currently ongoing. The results of phase I of this phase I/II study were reported at the ASCO 2017 with favorable outcomes (ORR of $25.0 \%$ in 40 cases) [9]; the eagerly awaited phase II is expected to be completed in April 2018.

\section{Combination of Immune Checkpoint Inhibitors and Molecular Targeted Therapy}

The therapeutic outcomes of nivolumab plus ipilimumab are superior to those of monotherapy in melanoma $[6,10]$. Therapy involving an immune checkpoint inhibitor plus a molecular targeted agent was suggested as a promising strategy in recent years. In HCC, interstitial cells (Kupffer cells, dendritic cells, liver endothelial cells, and liver stellate cells) and immunosuppressive cytokines (e.g., IL-10 or TGF- $\beta$ ) may contribute to the immunosuppressive environment, and the PD-1/PD-L1 pathway plays an important role in the development of the immunosuppressive microenvironment in HCC. Combining a molecular targeted agent and an immune checkpoint inhibitor is expected to improve this immunosuppressive microenvironment [11] (Fig. 2).

Table 3 shows the currently ongoing trials evaluating combination therapy involving immune checkpoint inhibition with molecular targeted therapy. A trial evaluating the combination of pembrolizumab and lenvatinib for the treatment of HCC was started in Japan and 
Table 3. Immune checkpoint inhibitors in combination with tyrosine kinase inhibitors in hepatocellular carcinoma

\begin{tabular}{lllll}
\hline Phase & Target & Agent & Company & Trial \# \\
\hline $1-2$ & PD-1 + TGF- $\beta$ receptor I & Nivolumab + galunisertib (LY2157299) & Eli Lilly & NCT02423343 \\
1 & PD-L1 + VEGFR-2 & Ramucirumab + durvalumab (MEDI4736) & Eli Lilly & NCT02572687 \\
1 & PD-1 + multikinase & Pembrolizumab + lenvatinib & Eisai & NCT03006926 \\
1 & PD-1 + multikinase & Pembrolizumab + nintedanib & Gustave Roussy & NCT02856425 \\
1 & PD-1 + multikinase & PDR001 + sorafenib & Novartis & NCT02988440 \\
$1-2$ & PD-1 + c-Met & PDR001 + capmatinib (INC280) & Novartis & NCT02795429 \\
$1-2$ & PD-1 + CTLA-4 + MET/VEGFR2 & Nivolumab + ipilimumab + cabozantinib & BMS & NCT01658878 \\
1 & PD-1 + multikinase & Nivolumab + lenvatinib & Ono & NCT03289533 \\
$1-2$ & PD-L1 + multikinase & Avelumab + axitinib & Pfizer & Roche \\
$1-2$ & PD-L1 + multikinase & Atezolizumab + bevacizumab & & \\
\hline
\end{tabular}

then will be expanded to the rest of the world. High response rates (50-70\%) and the longlasting durable response of this combination therapy in other types of solid cancer (e.g., kidney cancer and endometrial cancer) were presented at the ESMO 2016 and ASCO 2017. Therefore, similar high response rates and long-lasting durable responses are highly expected in HCC as well.

The mechanism underlying the synergistic effect of an immune checkpoint inhibitor plus a molecular targeted agent, unlike that of the combination of two immune checkpoint inhibitors, needs to be thoroughly clarified. Regarding the mechanism of action of pembrolizumablenvatinib combination therapy [12], a preclinical study including in vitro and in vivo studies showed that suppression of tumor-associated macrophages, regulatory $\mathrm{T}$ cells, and other constituents of the tumor-suppressive microenvironment resulted in decreases in TGF- $\beta$ and IL-10, the downregulation of PD-1 and Tim3, and the upregulation of ICOS and OX40, thereby inducing tumor immunity through IL-12 [13] (Fig. 2). It is anticipated that similar future studies on HCC will identify the best combination between a specific immune checkpoint inhibitor and molecular targeted agent. In addition to pembrolizumab-lenvatinib combination therapy (Fig. 3), many similar combination therapies for HCC are currently being evaluated in early-phase clinical trials (Table 3).

\section{Combination of Immune Checkpoint Inhibitors with an Existing Locoregional Therapy}

A different approach, namely, combining an immune checkpoint inhibitor with an existing locoregional therapy for HCC, is currently under evaluation. Transcatheter arterial chemoembolization (TACE), radiofrequency ablation (RFA), or radiation therapy is expected to enhance the effects of immunotherapy by inducing local inflammation. This would lead to the release of neoantigens that activate antigen presentation and the relevant immune system activation. These locoregional therapies are particularly beneficial when the levels of cancer antigens are negligible because of poor antigen release. The results of combination therapy with an anti-CTLA-4 antibody and locoregional therapy in advanced HCC were recently published [14]. The NCT01853618 study evaluated the efficacy of adjuvant therapy with tremelimumab (anti-CTLA-4 antibody) after RFA or TACE in several, but not all, HCC nodules, with favorable outcomes, including a partial response rate of $26 \%$, time to tumor progression of 7.4 months, and overall survival of 12.3 months. Increases in $\mathrm{CD}^{+}$and $\mathrm{CD}^{+}$cells in untreated nodules were clearly confirmed and attributed to the abscopal effect. 
DLT evaluation part

- $N=6-10$

- Starting dose of lenvatinib: $12 \mathrm{mg}$ QD (BW $\geq 60 \mathrm{~kg}) / 8 \mathrm{mg}$ QD $(\mathrm{BW}<60 \mathrm{~kg}$ )

- Dose of pembrolizumab: $200 \mathrm{mg} / \mathrm{Q} 3 \mathrm{~W}$

- BCLC: Stage B or C

- Child-Pugh A

- ECOG PS: 0-1

Study Start Date: January 2017

Estimated Study Completion Date: June 2019

Estimated Primary Completion Date: December 2018

(Final data collection date for primary outcome measure)

JapicCTI-173494. NCT03006926

Fig. 3. Phase Ib study of lenvatinib plus pembrolizumab in hepatocellular carcinoma.

\section{Phase II, exploratory study, Japan}
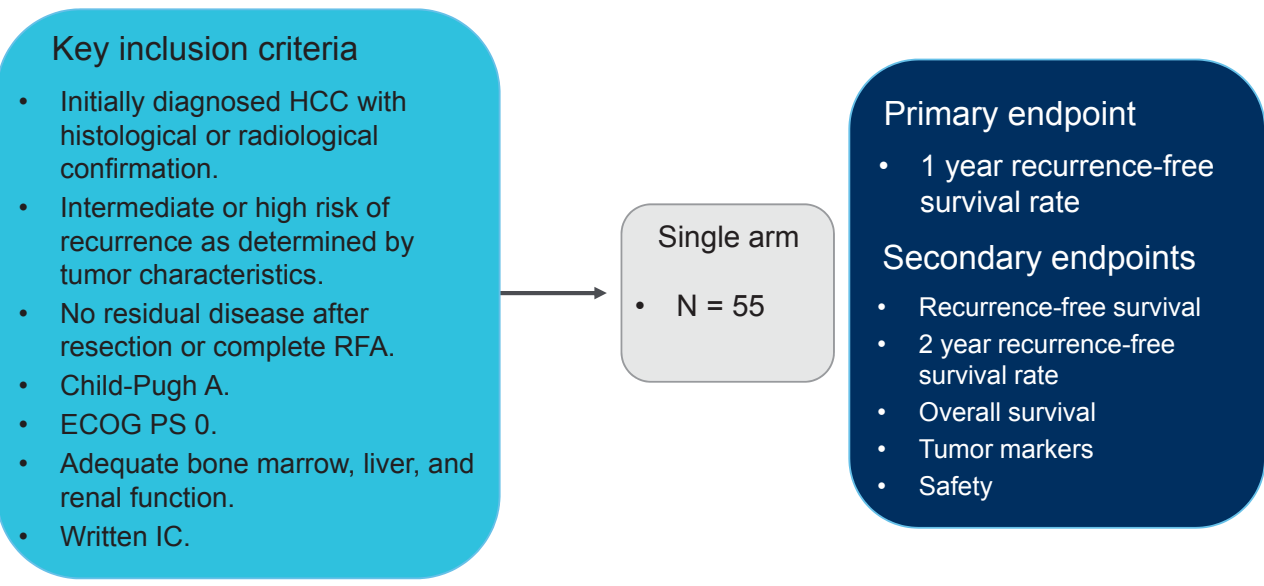

Study Start Date: Feb 2017

PI: Masatoshi Kudo (Kindai University)

UMIN000026648

Fig. 4. Adjuvant treatment with anti-PD-1 antibody to prevent recurrence after curative treatment of HCC. HCC, hepatocellular carcinoma; RFA, radiofrequency ablation; ECOG PS, Eastern Cooperative Oncology Group Performance Status; IC, informed consent.

Immune checkpoint inhibitors (antibodies to PD-1, PD-L1, and CTLA-4) are potentially beneficial in all forms of neoadjuvant therapy, adjuvant therapy after resection or ablation, and in combination with TACE, cytotoxic chemotherapy, or radiotherapy (Fig. 1).

HCC recurrence rates after curative therapy (resection or ablation) are particularly high, and its management remains an unmet need. This is mainly because of the presence of 


\section{Liver Cancer}

Fig. 5. Improved overall survival as a result of combination therapy: very near future. MST, median survival time.

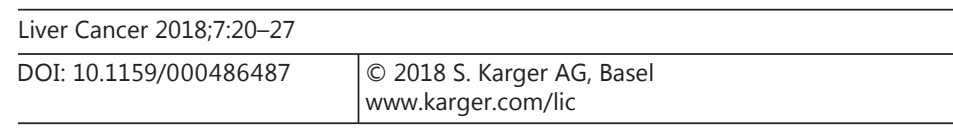

Kudo: Combination Cancer Immunotherapy in Hepatocellular Carcinoma

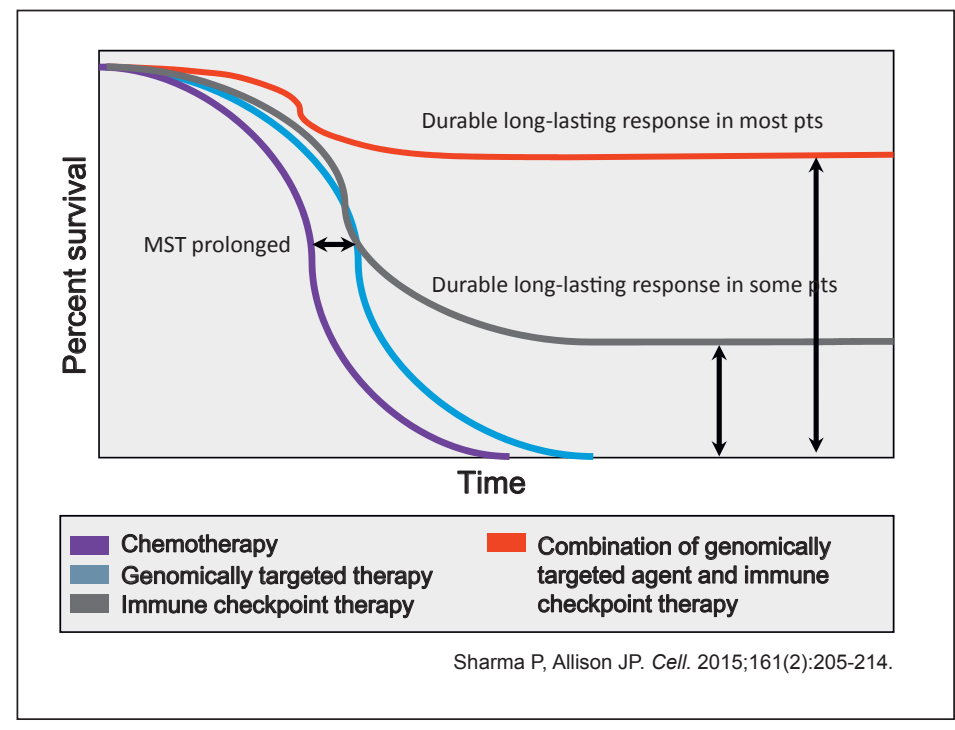

extremely small microsatellite metastatic lesions that are undetectable by imaging even at the time of resection or ablation. To address this issue, various agents (e.g., IFN, peretinoin, vitamin $\mathrm{K}$, and sorafenib) were tested for their efficacy as an adjuvant therapy in clinical trials, although all showed negative results [15-18].

Theoretically, microsatellite lesions and intrahepatic metastases may be suppressed by administration of anti-PD-1 antibody after recruitment of cytotoxic T lymphocytes to the microsatellite lesions upon release of tumor antigens by TACE or RFA [19]. Mizukoshi et al. [20] observed a significant increase in tumor-specific T cells, which is indicative of posttreatment tumor antigen release, after RFA in $62 \%$ of patients, and a significant correlation between tumor-specific $T$ cells and recurrence-free survival.

A clinical trial of nivolumab in the adjuvant setting after curative treatment was started in February 2017 in Japan (Fig. 4). The combination strategy of an immune checkpoint inhibitor in the adjuvant setting with curative therapy (resection or RFA) is anticipated to prevent HCC recurrence effectively.

\section{Conclusion}

Padmanee Sharma and James P. Allison predicted the possibility of long-term survival and real cure in patients who responded to combination treatment with immune checkpoint inhibitors and molecular targeted agents because its high efficacy can lead to "cure in a real sense" [21, 22] (Fig. 5). Indeed, immune checkpoint inhibitors will extend the overall survival of HCC patients, and, further, combination therapy with molecular targeted agents may result in real cure, which might lead to a paradigm shift in the treatment of HCC.

Emerging therapeutic strategies involving immune checkpoint inhibition combined with other treatment modalities will definitely change the future landscape of HCC treatment. 
Kudo: Combination Cancer Immunotherapy in Hepatocellular Carcinoma

\section{References}

1 Wolchok JD, Kluger H, Callahan MK, Postow MA, Rizvi NA, Lesokhin AM, Segal NH, et al: Nivolumab plus ipilimumab in advanced melanoma. N Engl J Med 2013;369:122-133.

2 Ansell SM, Lesokhin AM, Borrello I, Halwani A, Scott EC, Gutierrez M, Schuster SJ, et al: PD-1 blockade with nivolumab in relapsed or refractory Hodgkin's lymphoma. N Engl J Med 2015;372:311-319.

3 Robert C, Schachter J, Long GV, Arance A, Grob JJ, Mortier L, Daud A, et al: Pembrolizumab versus ipilimumab in advanced melanoma. N Engl J Med 2015;372:2521-2532.

4 Garon EB, Rizvi NA, Hui R, Leighl N, Balmanoukian AS, Eder JP, Patnaik A, et al: Pembrolizumab for the treatment of non-small-cell lung cancer. N Engl J Med 2015;372:2018-2028.

5 Brahmer J, Reckamp KL, Baas P, Crino L, Eberhardt WE, Poddubskaya E, Antonia S, et al: Nivolumab versus docetaxel in advanced squamous-cell non-small-cell lung cancer. N Engl J Med 2015;373:123-135.

6 Larkin J, Chiarion-Sileni V, Gonzalez R, Grob JJ, Cowey CL, Lao CD, Schadendorf D, et al: Combined nivolumab and ipilimumab or monotherapy in untreated melanoma. N Engl J Med 2015;373:23-34.

7 El-Khoueiry AB, Sangro B, Yau T, Crocenzi TS, Kudo M, Hsu C, Kim TY, et al: Nivolumab in patients with advanced hepatocellular carcinoma (CheckMate 040): an open-label, non-comparative, phase 1/2 dose escalation and expansion trial. Lancet 2017;389:2492-2502.

8 Kudo M: Molecular targeted agents for hepatocellular carcinoma: current status and future perspectives. Liver Cancer 2017;6:101-112.

9 Kelley RK, Abou-Alfa GK, Bendell JC, Kim TY, Borad MJ, Yong WP, Morse M, et al: Phase I/II study of durvalumab and tremelimumab in patients with unresectable hepatocellular carcinoma (HCC): phase I safety and efficacy analyses. J Clin Oncolol 2017;35(suppl); abstract 4073.

10 Postow MA, Chesney J, Pavlick AC, Robert C, Grossmann K, McDermott D, Linette GP, et al: Nivolumab and ipilimumab versus ipilimumab in untreated melanoma. N Engl J Med 2015;372:2006-2017.

11 Tiegs G, Lohse AW: Immune tolerance: what is unique about the liver. J Autoimmun 2010;34:1-6.

12 Kudo M, Finn RS, Qin S, Han KH, Ikeda K, Piscaglia F, Baron A, et al: A randomised phase 3 trial of lenvatinib vs sorafenib in first-line treatment of patients with unresectable hepatocellular carcinoma. Lancet 2017, in press.

13 Kato T, Bao X, Macgrath S, Tabata K, Hori Y, Tachino S, Matijevici M, et al: Lenvatinib mesilate (LEN) enhanced antitumor activity of a PD-1 blockade agent by potentiating Th1 immune response. Ann Oncol 2016;27:1-14

14 Duffy AG, Ulahannan SV, Makorova-Rusher O, Rahma O, Wedemeyer H, Pratt D, Davis JL, et al: Tremelimumab in combination with ablation in patients with advanced hepatocellular carcinoma. J Hepatol 2017;66:545551.

15 Yoshida H, Shiratori Y, Kudo M, Shiina S, Mizuta T, Kojiro M, Yamamoto K, et al: Effect of vitamin K2 on the recurrence of hepatocellular carcinoma. Hepatology 2011;54:532-540.

16 Bruix J, Takayama T, Mazzaferro V, Chau GY, Yang J, Kudo M, Cai J, et al: Adjuvant sorafenib for hepatocellular carcinoma after resection or ablation (STORM): a phase 3, randomised, double-blind, placebo-controlled trial. Lancet Oncol 2015;16:1344-1354.

17 Mazzaferro V, Romito R, Schiavo M, Mariani L, Camerini T, Bhoori S, Capussotti L, et al: Prevention of hepatocellular carcinoma recurrence with alpha-interferon after liver resection in HCV cirrhosis. Hepatology 2006; 44:1543-1554.

18 Okita K, Izumi N, Matsui O, Tanaka K, Kaneko S, Moriwaki H, Ikeda K, et al: Peretinoin after curative therapy of hepatitis C-related hepatocellular carcinoma: a randomized double-blind placebo-controlled study. J Gastroenterol 2015;50:191-202.

19 Kudo M: Immune checkpoint inhibition in hepatocellular carcinoma: basics and ongoing clinical trials. Oncology 2017;92(suppl 1):50-62.

20 Mizukoshi E, Yamashita T, Arai K, Sunagozaka H, Ueda T, Arihara F, Kagaya T, et al: Enhancement of tumorassociated antigen-specific T cell responses by radiofrequency ablation of hepatocellular carcinoma. Hepatology 2013;57:1448-1457.

21 Sharma P, Allison JP: Immune checkpoint targeting in cancer therapy: toward combination strategies with curative potential. Cell 2015;161:205-214.

22 Sharma P, Allison JP: The future of immune checkpoint therapy. Science 2015;348:56-61. 